\title{
Unravelling radiative energy transfer in solid-state lighting
}

Rustamzhon Melikov, Daniel Aaron Press, Baskaran Ganesh Kumar, Sadra Sadeghi, and Sedat Nizamoglu

Citation: Journal of Applied Physics 123, 023103 (2018); doi: 10.1063/1.5008922

View online: https://doi.org/10.1063/1.5008922

View Table of Contents: http://aip.scitation.org/toc/jap/123/2

Published by the American Institute of Physics

\section{Articles you may be interested in}

Addressing limitations of photoluminescence based external quantum efficiency measurements Journal of Applied Physics 123, 023105 (2018); 10.1063/1.5004193

Photonic nanojet assisted enhancement of Raman signal: Effect of refractive index contrast Journal of Applied Physics 123, 023102 (2018); 10.1063/1.4994944

Optical nutation in acetylene-filled hollow-core photonic crystal fiber Journal of Applied Physics 123, 023101 (2018); 10.1063/1.5004179

Thermoacoustics of solids: A pathway to solid state engines and refrigerators Journal of Applied Physics 123, 024903 (2018); 10.1063/1.5006489

Internal photoemission for photovoltaic using p-type Schottky barrier: Band structure dependence and theoretical efficiency limits

Journal of Applied Physics 123, 023107 (2018); 10.1063/1.5003117

Compositional and strain analysis of $\operatorname{In}(\mathrm{Ga}) \mathrm{N} / \mathrm{GaN}$ short period superlattices

Journal of Applied Physics 123, 024304 (2018); 10.1063/1.5009060

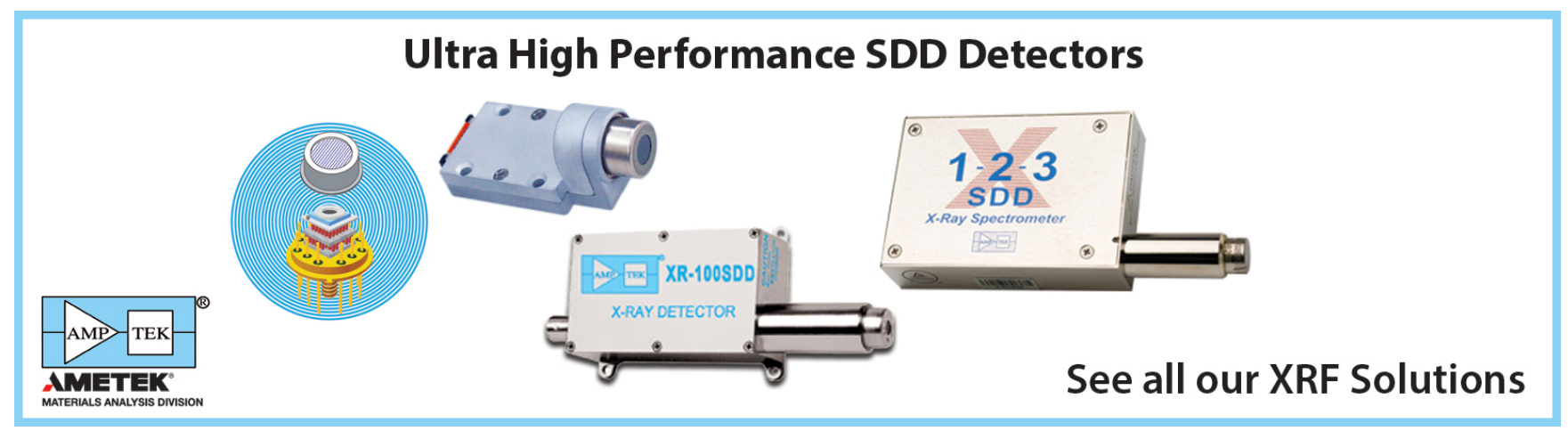




\title{
Unravelling radiative energy transfer in solid-state lighting
}

\author{
Rustamzhon Melikov, ${ }^{1, a)}$ Daniel Aaron Press, ${ }^{1, a)}$ Baskaran Ganesh Kumar, ${ }^{1}$ \\ Sadra Sadeghi, ${ }^{2}$ and Sedat Nizamoglu ${ }^{1,2,3, b)}$ \\ ${ }^{1}$ Department of Electrical and Electronics Engineering, Koc University, 34450 Sariyer, Istanbul, Turkey \\ ${ }^{2}$ Graduate School of Materials Science and Engineering, Koc University, 34450 Sariyer, Istanbul, Turkey \\ ${ }^{3}$ Graduate School of Biomedical Sciences and Engineering, Koc University, 34450 Sariyer, Istanbul, Turkey
}

(Received 11 October 2017; accepted 18 December 2017; published online 9 January 2018)

\begin{abstract}
Today, a wide variety of organic and inorganic luminescent materials (e.g., phosphors, quantum dots, etc.) are being used for lighting and new materials (e.g., graphene, perovskite, etc.) are currently under investigation. However, the understanding of radiative energy transfer is limited, even though it is critical to understand and improve the performance levels of solid-state lighting devices. In this study, we derived a matrix approach that includes absorption, reabsorption, interabsorption and their iterative and combinatorial interactions for one and multiple types of fluorophores, which is simplified to an analytical matrix. This mathematical approach gives results that agree well with the measured spectral and efficiency characteristics of color-conversion light-emitting diodes. Moreover, it also provides a deep physical insight by uncovering the entire radiative interactions and their contribution to the output optical spectrum. The model is universal and applicable for all kinds of fluorophores. Published by AIP Publishing. https://doi.org/10.1063/1.5008922
\end{abstract}

\section{INTRODUCTION}

Today, solid-state lighting has become an integral technology that we use in our TVs, mobile phones, cars, homes and offices due to its efficiency, compactness, lifetime and robustness. ${ }^{1}$ In this technology, blue or UV light-emitting diode (LED) chips emit light by electroluminescence, and the color conversion material, which is integrated on top of the LED die, is pumped by the absorption of electroluminescence. The combined electroluminescence and Stokesshifted spontaneous emission by the fluorophore is detected as white color by the human eye. Single, double, and triple combinations of these luminescent materials with distinct spectral properties are commonly used. The observed white emission spectrum is generated via radiative energy transfer due to absorption of electroluminescence, absorption of photoluminescence by the same type of fluorophore (reabsorption) and absorption of photoluminescence by different types of fluorophores (inter-absorption). Until the extraction of the photons from the luminescent material, the photons have an infinite number of possible radiative interactions in the color conversion material. $^{2}$

Today, dyes, polymers, phosphors and quantum dots are widely used for lighting and display applications; there are many new materials, including fluorescent proteins (FPs), perovskite and graphene, finding applications. ${ }^{3-11}$ There are an unlimited number of possible combinations of materials and their subsets, each with distinct optical properties. Predicting and understanding the possible optical properties are important for solid-state lighting. In general, previous numerical studies have combined distinct emission profiles by varying their optical properties such as peaks, central wavelengths and full width at half maxima (FWHMs) to

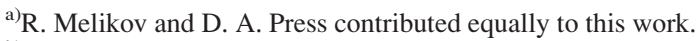

${ }^{\text {b)} E-m a i l: ~ s n i z a m o g l u @ k u . e d u . t r ~}$
}

predict the approximate photometric properties of solid-state lighting technologies. ${ }^{12-15}$ These studies have estimated the approximate spectral properties without considering the detailed photonic processes occurring.

In this study, we developed a matrix method that generates the output spectrum of down-conversion LEDs by including radiative energy transfer processes. We derived the algebra, including all the possible optical interactions of absorption, reabsorption and inter-absorption for one and multiple types of fluorophores. This algebraic expression was then simplified to an analytical matrix. This method predicts the experimental LED spectrum and resolves the individual contributions to the output spectrum from different processes. To show the applicability of the method, we experimentally measured the performance of FP based LEDs and compared them with computational results. The theoretical and experimental results show good agreement.

\section{THEORY}

Radiative energy transfer is based on two fundamental processes of photon absorption and spontaneous emission. For this process to occur, the absorption spectrum of the fluorophore and the emission spectrum of the pump need to overlap. The incoming photon, which has a higher energy, is absorbed and excites the molecule. The molecules in an excited state can then possibly decay via a radiative recombination [see Fig. 1(a)]. As a bulk process, when the incoming photon flux (i.e., blue electroluminescence in white LEDs) with a specific power spectral density $\left(S_{i n}(\lambda)\right)$ interacts with a fluorophore (having absorption coefficient $\alpha(\lambda)$ ), this interaction results in photoluminescence $(P(\lambda))$. The unabsorbed power spectral density of the pump transmits through the fluorophore and combines with the photoluminescence of the fluorophore to generate light with a total power spectral density (of $S_{\text {out }}^{A}(\lambda)$ ) [see Fig. 1(b)]. The left part of Eq. (1) is the 

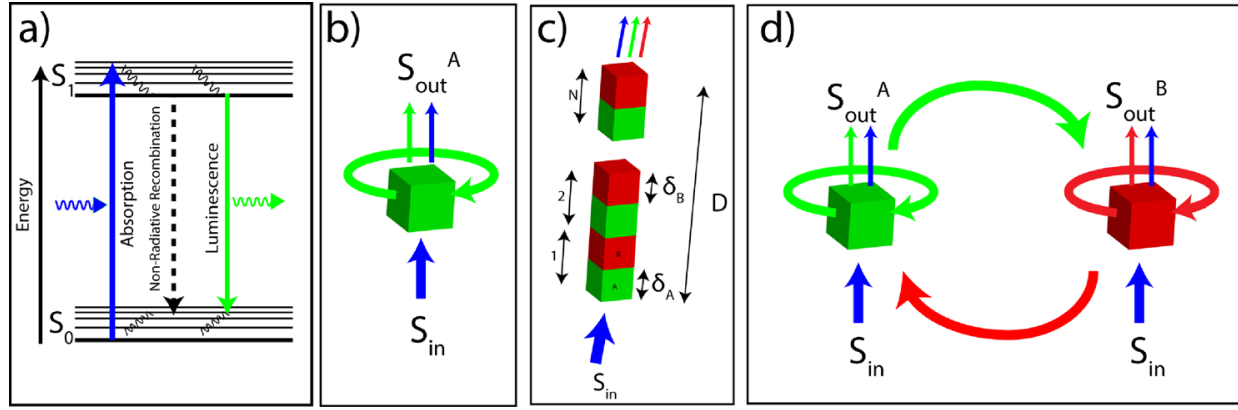

FIG. 1. (a) Jablonski diagram for luminescence. $\mathrm{S}_{0}$ : ground states, $\mathrm{S}_{1}$ : excited states. The schematic representation of color conversion for (b) one and (d) a mixture of two types of fluorophores. (c) Schematic of a homogeneous mixture of two fluorophores, which is modeled as $\mathrm{N}$ stacks with thicknesses of $\delta_{A}$ and $\delta_{B}$. Green box: fluorophore A; red box: fluorophore B; blue arrow: electroluminescence of the pumping LED; $\mathrm{S}_{\text {in: }}$ power spectral density of electroluminescence; circular arrow: reabsorption; arrow between fluorophores: inter-absorption; $\mathrm{S}_{\text {out }}{ }^{\mathrm{x}}$ : the generated output power spectral density (including transmitted electroluminescence and photoluminescence) by the fluorophore $\mathrm{x}(\mathrm{x}=\mathrm{A}$ and $\mathrm{B})$.

transmitted pump light through the fluorophore and the right part of the equation is the emitted light from the fluorophore, where $P(\lambda)$ is the normalized photoluminescence spectrum and $\mathrm{W}$ is [see Eq. (2)] the emission strength, which is proportional to the amount of absorbed light by the fluorophore integrated over the spectrum and scaled by quantum yield. ${ }^{16}$ When absorption and emission spectra of the fluorophore overlap, reabsorption occurs; hence, in the second part, the multiplication term $\mathrm{C}(\lambda)$ is the contribution to the overall emission due to reabsorption, which is the sum of infinitely many cycles of reabsorption [see Eq. (3)]. The reabsorption parameter $\mathrm{f}^{\mathrm{n}}$ is the amount of reabsorbed light for the $\mathrm{n}^{\text {th }}$ cycle, which is further integrated over the spectrum and scaled by quantum yield [see Eq. (4)]. When there is no reabsorption $(f=0)$, the $C(\lambda)$ factor approaches unity. Here, we assume that the physical path length (D) is equal to the effective optical path length.

Our calculation is based on Eqs. (1) and (2), ${ }^{16}$ in which $\mathrm{C}(\lambda)$ is the responsible variable that includes the reabsorption processes for one type of fluorophore. Here, it is assumed that an incident photon (generated by a fluorophore) is directly reabsorbed by another fluorophore, which is in the ground state. ${ }^{17-19}$ This is a proper assumption in our case as well because in LEDs, the fluorophore excitation and light emission are not saturated at moderate current injection levels. Thus, each absorbed photon brings the molecule to an excited state. Therefore, $C(\lambda)$ parameter, which shows absorptionreabsorption cycles, does not contain any timescale for radiative energy transfer processes.

$$
\begin{aligned}
S_{\text {out }}(\lambda) & =S_{\text {in }}(\lambda) \exp [-\alpha(\lambda) D]+W C(\lambda) P(\lambda), \\
W & =Q \int S_{\text {in }}(\lambda)\{1-\exp [-\alpha(\lambda) D]\} d \lambda, \\
C(\lambda) & =\left[\sum_{n=0}^{\infty} \mathrm{f}^{n}\right] \exp [-\alpha(\lambda) D] \\
& =\frac{\exp [-\alpha(\lambda) D]}{1-Q \int P(\lambda)\{1-\exp [-\alpha(\lambda) D]\} d \lambda}, \\
f & =Q \int P(\lambda)\{1-\exp [-\alpha(\lambda) D]\} d \lambda .
\end{aligned}
$$

For a mixture of two types of fluorophores, it is important to identify the ratio of pump electroluminescence that is absorbed by each fluorophore $(\kappa)$. For that, a homogenous mixture of fluorophores is considered [see Fig. 1(c)], and $\kappa$ for each fluorophore is defined in Eqs. (5) and (6) [see supplementary material for derivation]

$$
\begin{gathered}
\kappa_{\mathrm{A}}(\lambda)=\frac{\alpha_{\mathrm{A}}(\lambda) \mathrm{D}_{\mathrm{A}}}{\alpha_{\mathrm{A}}(\lambda) \mathrm{D}_{\mathrm{A}}+\alpha_{\mathrm{B}}(\lambda) \mathrm{D}_{\mathrm{B}}}, \\
\kappa_{B}(\lambda)=\frac{\alpha_{B}(\lambda) D_{B}}{\alpha_{A}(\lambda) D_{A}+\alpha_{B}(\lambda) D_{B}} .
\end{gathered}
$$

In a mixture of two types of fluorophores, the output light spectrum consists of the transmitted electroluminescence through the fluorophores, photoluminescence due to absorption of electroluminescence, and photoluminescence due to reabsorption and inter-absorption. Each fluorophore can excite the other type of fluorophore and in this situation inter-absorption occurs, in which fluorophore $\mathrm{A}$ can excite fluorophore $\mathrm{B}\left(\mathrm{f}_{\mathrm{AB}}\right)$ and fluorophore $\mathrm{B}$ can also excite fluorophore $\mathrm{A}\left(\mathrm{f}_{\mathrm{BA}}\right)$. Consequently, these inter-absorbed photons can recombine radiatively and generate photoluminescence. Furthermore, in the process of reabsorption, the photoluminescence by a fluorophore can be absorbed by the same type of fluorophores $\left(\mathrm{f}_{\mathrm{AA}}\right.$ and $\mathrm{f}_{\mathrm{BB}}$ ) and these reabsorbed photons can have radiative decay and generate photoluminescence. The emission due to reabsorption or inter-absorption can again go through reabsorption or inter-absorption and generate photoluminescence. This pumping due to reabsorption and interabsorption can take place infinitely many times [see Fig. 1(d) for possible interactions], and consequently, Eq. (7) includes all the possible iterations [see Eqs. (8)-(13) for the mathematical formula of the variables in Eq. (7) ${ }^{20}$

$$
\begin{aligned}
S_{\text {out }}(\lambda)= & S_{\text {in }}(\lambda) \exp \left[-\left(\alpha_{A}(\lambda) D_{A}+\alpha_{B}(\lambda) D_{B}\right)\right] \\
& +W_{A} P_{A}(\lambda) \exp \left[-\left(\alpha_{A}(\lambda) D_{A}+\alpha_{B}(\lambda) D_{B}\right)\right] \\
& +W_{B} P_{B}(\lambda) \exp \left[-\left(\alpha_{A}(\lambda) D_{A}+\alpha_{B}(\lambda) D_{B}\right)\right] \\
& +W_{A} f_{A A}(\lambda) P_{A}(\lambda) \exp \left[-\left(\alpha_{A}(\lambda) D_{A}+\alpha_{B}(\lambda) D_{B}\right)\right] \\
& +W_{A} f_{A B}(\lambda) P_{B}(\lambda) \exp \left[-\left(\alpha_{A}(\lambda) D_{A}+\alpha_{B}(\lambda) D_{B}\right)\right] \\
& +W_{B} f_{B A}(\lambda) P_{A}(\lambda) \exp \left[-\left(\alpha_{A}(\lambda) D_{A}+\alpha_{B}(\lambda) D_{B}\right)\right] \\
& +W_{B} f_{B B}(\lambda) P_{B}(\lambda) \exp \left[-\left(\alpha_{A}(\lambda) D_{A}+\alpha_{B}(\lambda) D_{B}\right)\right] \\
& +\cdots,
\end{aligned}
$$


$W_{A}=Q_{A} \int S_{i n}(\lambda) \kappa_{A}(\lambda)\left\{1-\exp \left[-\left(\alpha_{A}(\lambda) D_{A}+\alpha_{B}(\lambda) D_{B}\right)\right]\right\} d \lambda$,

$W_{B}=Q_{B} \int S_{i n}(\lambda) \kappa_{B}(\lambda)\left\{1-\exp \left[-\left(\alpha_{A}(\lambda) D_{A}+\alpha_{B}(\lambda) D_{B}\right)\right]\right\} d \lambda$,

$f_{A A}=Q_{A} \int P_{A}(\lambda) \kappa_{A}(\lambda)\left\{1-\exp \left[-\left(\alpha_{A}(\lambda) D_{A}+\alpha_{B}(\lambda) D_{B}\right)\right]\right\} d \lambda$,

$f_{A B}=Q_{B} \int P_{A}(\lambda) \kappa_{B}(\lambda)\left\{1-\exp \left[-\left(\alpha_{A}(\lambda) D_{A}+\alpha_{B}(\lambda) D_{B}\right)\right]\right\} d \lambda$,

$f_{B A}=Q_{A} \int P_{B}(\lambda) \kappa_{A}(\lambda)\left\{1-\exp \left[-\left(\alpha_{A}(\lambda) D_{A}+\alpha_{B}(\lambda) D_{B}\right)\right]\right\} d \lambda$,

$f_{B B}=Q_{B} \int P_{B}(\lambda) \kappa_{B}(\lambda)\left\{1-\exp \left[-\left(\alpha_{A}(\lambda) D_{A}+\alpha_{B}(\lambda) D_{B}\right)\right]\right\} d \lambda$.

To simplify the calculation on two fluorophore color conversion, a $2 \times 2$ matrix [see Eq. (14)], which can explain all the radiative energy transfer interactions, is introduced (see Table I). $\bar{M}^{0}$ corresponds to the matrix with zero cycle, which means without any reabsorption (R) or inter-absorption (I) process, hence, the non-diagonal terms are zero due to no reabsorption and interabsorption. $\overline{\bar{M}}^{1}$ corresponds to one cycle of inter-absorption or reabsorption (i.e.,, possible events are abbreviated as $\mathrm{R}$ or I), and with this iteration, the nondiagonal terms start to be non-zero. $\overline{\bar{M}}^{2}$ corresponds to 2 cycles of reabsorption or inter-absorption (i.e., successive possible events are RR, II, IR, RI). Hence, $\overline{\bar{M}}^{\mathrm{n}}$ corresponds to $\mathrm{n}$ number of reabsorption or inter-absorption cycles, which has $2^{\text {n+1 }}$ summing elements. For example, in $\overline{\bar{M}}^{3}$, the $\mathrm{M}_{11}$ element consists of 4 elements: $\mathrm{f}_{\mathrm{AA}} \mathrm{f}_{\mathrm{AA}} \mathrm{f}_{\mathrm{AA}}+\mathrm{f}_{\mathrm{AB}} \mathrm{f}_{\mathrm{BA}}$ $\mathrm{f}_{\mathrm{AA}}+\mathrm{f}_{\mathrm{AA}} \mathrm{f}_{\mathrm{AB}} \mathrm{f}_{\mathrm{BA}}+\mathrm{f}_{\mathrm{AB}} \mathrm{f}_{\mathrm{BB}} \mathrm{f}_{\mathrm{BA}}$ and each component in $\overline{\bar{M}}^{3}$ consists of 4 summation elements; thus, this $2 \times 2$ matrix has 16 summation elements, in total. The summation of $\overline{\bar{M}}$ matrices consecutively multiplied by itself results in an increasing number of elements [see Eqs. (S24)-(S27)] (supplementary material). Thus, the number of summations is increasing (e.g., $\overline{\bar{M}}^{4}$ has 32 and $\overline{\bar{M}}^{5}$ has 64 terms), but each higher order contributes lower and lower to the overall emission. Hence, the infinite sum of these interactions is written in the simplified form [see Eq. (15)], which yields to the output color conversion spectrum [see Eq. (16)]

$$
\begin{gathered}
\overline{\bar{M}}=\left|\begin{array}{ll}
M_{11} & M_{12} \\
M_{21} & M_{22}
\end{array}\right| \\
\overline{\bar{C}}=\left(\overline{\bar{M}}^{0}+\overline{\bar{M}}^{1}+\overline{\bar{M}}^{2}+\overline{\bar{M}}^{3}+\cdots\right) \exp \left[-\sum_{j} \alpha_{j}(\lambda) D_{j}\right] \\
=\sum_{n=0}^{\infty} \overline{\bar{M}}^{n} \exp \left[-\sum_{j} \alpha_{j}(\lambda) D_{j}\right]=(I-\overline{\bar{M}})^{-1} \\
\times \exp \left[-\sum_{j} \alpha_{j}(\lambda) D_{j}\right], \\
\overrightarrow{S_{\text {out }}}=\overrightarrow{S_{\text {in }}} \exp \left[-\sum_{j} \alpha_{j}(\lambda) D_{j}\right]+\vec{W}^{T} \times \overline{\bar{C}} \times \overline{\bar{P}} .
\end{gathered}
$$

Furthermore, the generalized equation forms of the output spectrum for an unlimited number of fluorophore interactions are shown in Eqs. (17)-(23) (see supplementary material for $\kappa_{i}(\lambda), W_{i}, \overline{\bar{M}}, \overline{\bar{P}}, \overline{\bar{C}}$ and $\overrightarrow{S_{\text {out }}}$ for mathematical description)

$$
\begin{aligned}
& \kappa_{i}(\lambda)=\frac{\alpha_{i}(\lambda) D_{i}}{\sum_{j} \alpha_{j}(\lambda) D_{j}}, \\
& W_{i}=Q_{i} \int S_{i n}(\lambda) \kappa_{i}(\lambda)\left\{1-\exp \left[-\sum_{j} \alpha_{j}(\lambda) D_{j}\right]\right\} d \lambda, \\
& \overline{\bar{M}}=\left(\begin{array}{ccc}
M_{1,1} & \cdots & M_{1, j} \\
\vdots & \ddots & \vdots \\
M_{i, 1} & \cdots & M_{i, j}
\end{array}\right) \text {, } \\
& M_{i, j}=Q_{j} \int P_{i}(\lambda) \kappa_{j}(\lambda)\left\{1-\exp \left[-\sum_{j} \alpha_{j}(\lambda) D_{j}\right]\right\} d \lambda, \\
& \overline{\bar{P}}=\left[\begin{array}{ccc}
P_{1,1} & \cdots & P_{1, n} \\
\vdots & \ddots & \vdots \\
P_{j, 1} & \cdots & P_{j, n}
\end{array}\right], \\
& \overline{\bar{C}}=(I-\overline{\bar{M}})^{-1} \exp \left[-\sum_{j} \alpha_{j}(\lambda) D_{j}\right] \text {, }
\end{aligned}
$$

TABLE I. Interaction matrix $\overline{\bar{M}}$ that reveals absorption, reabsorption and inter-absorption events of color conversion for a mixture of two types of fluorophores. $\overline{\bar{M}}^{\mathrm{n}}$ corresponds to the $\mathrm{n}$ number of absorption cycles $(\mathrm{n}=1,2, \ldots) . \mathrm{f}_{\mathrm{XY}}$ is the amount of absorbed light by fluorophore $\mathrm{X}$ from the emission of fluorophore $Y$ integrated over the spectrum and scaled by the quantum yield of fluorophore $X, Y(X, Y=A, B)$.

\begin{tabular}{lcccc}
\hline \hline$\overline{\bar{M}}$ & $\mathrm{M}_{11}$ & $\mathrm{M}_{12}$ & $\mathrm{M}_{21}$ & $\mathrm{M}_{22}$ \\
\hline$\overline{\bar{M}}^{0}$ & 1 & 0 & 0 & 1 \\
$\overline{\bar{M}}^{1}$ & $\mathrm{f}_{\mathrm{AA}}$ & $\mathrm{f}_{\mathrm{AB}}$ & $\mathrm{f}_{\mathrm{BA}}$ & $\mathrm{f}_{\mathrm{BB}}$ \\
$\overline{\bar{M}}^{2}$ & $\mathrm{f}_{\mathrm{AA}} \mathrm{f}_{\mathrm{AA}}+\mathrm{f}_{\mathrm{AB}} \mathrm{f}_{\mathrm{BA}}$ & $\mathrm{f}_{\mathrm{BA}} \mathrm{f}_{\mathrm{AA}}+\mathrm{f}_{\mathrm{BB}} \mathrm{f}_{\mathrm{BA}}$ & $\mathrm{f}_{\mathrm{BA}} \mathrm{f}_{\mathrm{AB}}+\mathrm{f}_{\mathrm{BB}} \mathrm{f}_{\mathrm{BB}}$ \\
$\overline{\bar{M}}^{3}$ & $\mathrm{f}_{\mathrm{AA}} \mathrm{f}_{\mathrm{AA}} \mathrm{f}_{\mathrm{AA}}+\mathrm{f}_{\mathrm{AB}} \mathrm{f}_{\mathrm{BA}} \mathrm{f}_{\mathrm{AA}}$ & $\mathrm{f}_{\mathrm{AA}} \mathrm{f}_{\mathrm{AB}}+\mathrm{f}_{\mathrm{AB}} \mathrm{f}_{\mathrm{BB}}$ & $\mathrm{f}_{\mathrm{AA}} \mathrm{f}_{\mathrm{AA}} \mathrm{f}_{\mathrm{AB}}+\mathrm{f}_{\mathrm{AB}} \mathrm{f}_{\mathrm{BA}} \mathrm{f}_{\mathrm{AB}}$ & $\mathrm{f}_{\mathrm{BA}} \mathrm{f}_{\mathrm{AA}} \mathrm{f}_{\mathrm{AB}}+\mathrm{f}_{\mathrm{BB}} \mathrm{f}_{\mathrm{BA}} \mathrm{f}_{\mathrm{AB}}$ \\
& $+\mathrm{f}_{\mathrm{AA}} \mathrm{f}_{\mathrm{AB}} \mathrm{f}_{\mathrm{BA}}+\mathrm{f}_{\mathrm{AB}} \mathrm{f}_{\mathrm{BB}} \mathrm{f}_{\mathrm{BA}}$ & $+\mathrm{f}_{\mathrm{BB}} \mathrm{f}_{\mathrm{BA}} \mathrm{f}_{\mathrm{AA}} \mathrm{f}_{\mathrm{AB}} \mathrm{f}_{\mathrm{BB}}+\mathrm{f}_{\mathrm{AB}} \mathrm{f}_{\mathrm{BB}} \mathrm{f}_{\mathrm{BB}}$ & $+\mathrm{f}_{\mathrm{BA}} \mathrm{f}_{\mathrm{AB}} \mathrm{f}_{\mathrm{BA}}+\mathrm{f}_{\mathrm{BB}} \mathrm{f}_{\mathrm{BB}} \mathrm{f}_{\mathrm{BA}}$ & $+\mathrm{f}_{\mathrm{BA}} \mathrm{f}_{\mathrm{AB}} \mathrm{f}_{\mathrm{BB}}+\mathrm{f}_{\mathrm{BB}} \mathrm{f}_{\mathrm{BB}} \mathrm{f}_{\mathrm{BB}}$ \\
$\cdots$ & $\cdots$ & $\cdots$ & $\cdots$ & $\cdots$ \\
\hline \hline
\end{tabular}




$$
\overrightarrow{S_{\text {out }}}=\overrightarrow{S_{\text {in }}} \exp \left[-\sum_{j} \alpha_{j}(\lambda) D_{j}\right]+\vec{W}^{T} \times \overline{\bar{C}} \times \overline{\bar{P}} .
$$

\section{RESULTS AND DISCUSSION}

\section{Simulation and experimental comparison of color conversion for one type of fluorescent protein}

To evaluate this model, we compared theoretical prediction with experimental observations. We selected FPs as our fluorophore because they have recently attracted attention for photonic devices due to their biocompatibility, bio-production capability and high spectral tunability. ${ }^{21,22}$ Moreover, the barrel-shaped shield of FPs protects the luminescent center from quenching against the photoinduced electron transfer. We biologically synthesized enhanced green FPs (eGFP) in an Escherichia coli expression system ${ }^{10}$ [see Fig. 2(a) for absorbance and photoluminescence of eGFP] and used eGFP as the test fluorophore. We placed eGFP solutions into a transparent hemispherical container (with an internal diameter of $4 \mathrm{~mm}$ ) on top of a blue LED chip, which has the peak electroluminescence wavelength at $448.5 \mathrm{~nm}$ with a FWHM of $17.1 \mathrm{~nm}$ [see Figs. S1(a) and S1(b)] (supplementary material).
The eGFP concentration on a LED was varied from $88.4 \mu \mathrm{M}$ up to $355.6 \mu \mathrm{M}$, which leads to sufficient interparticle distance to limit the possible Förster-type homotransfer interactions, and the absolute irradiances were measured by using an integrating sphere (Ocean Optics Torus spectrometer and FOIS-1 integrating sphere). For the simulation, we used the measured absorption and emission spectra shown in Fig. 2(a), and a quantum yield of 0.6 at a concentration of $35.2 \mu \mathrm{M}$. The simulated and measured results were visually in close agreement [see Fig. 2(b)]. There are many parameters that may influence the difference between simulated and experimental results such as refractive index mismatch, dispersion, total internal reflection, waveguiding effect and other possible optical loss mechanisms that exist in the optical system. To include possible loss mechanisms, the LED needs to be calibrated with the host material of fluorophores. For that, we calibrated our optical system, while the hemispherical lens contained water without any fluorescent protein [see Figs. S1(a) and S1(b)] (supplementary material).

To understand the effect of reabsorption processes, we analyzed the optical properties with/without a reabsorption process and compared them with the experimental results. When reabsorption is neglected, the external quantum efficiency stays a)

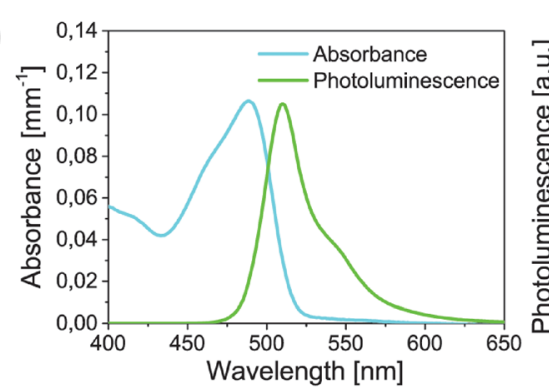

d)

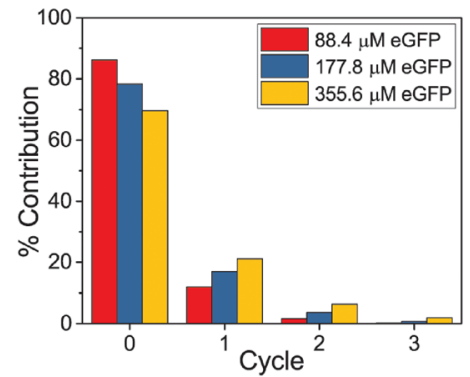

g)

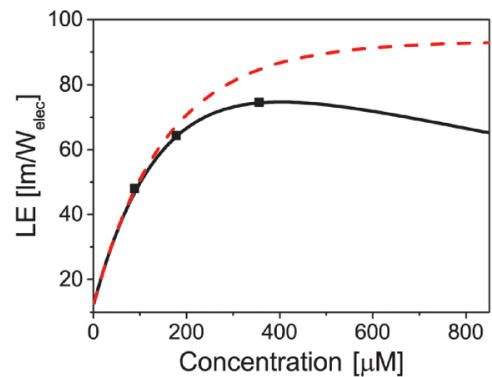

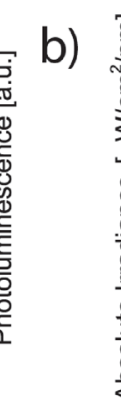

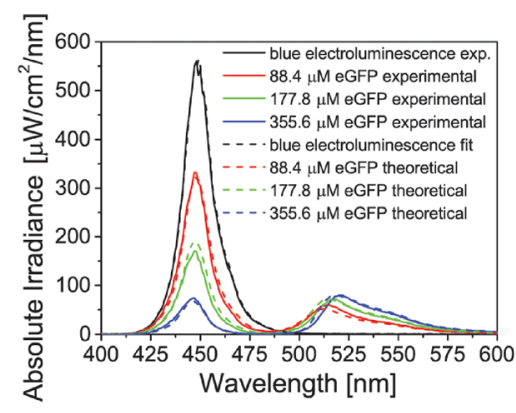

C)

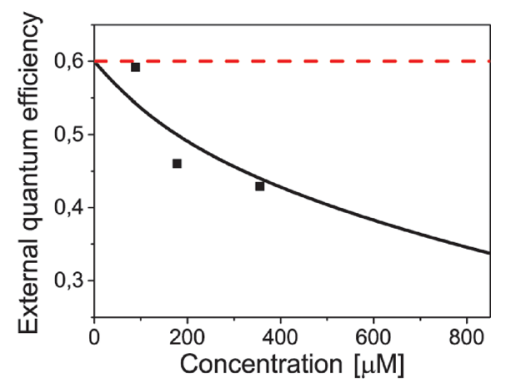

e)

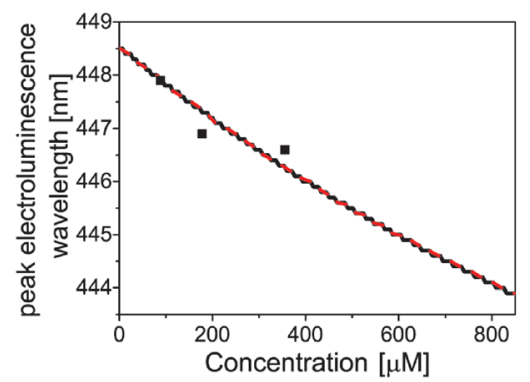

f)

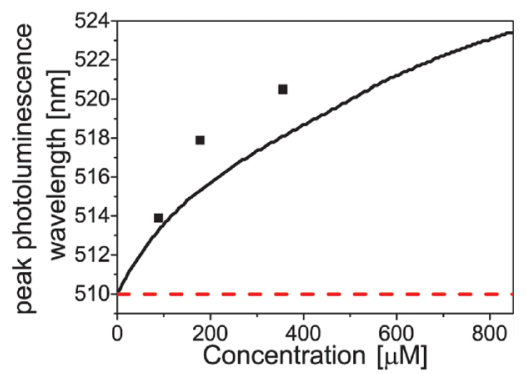

h)

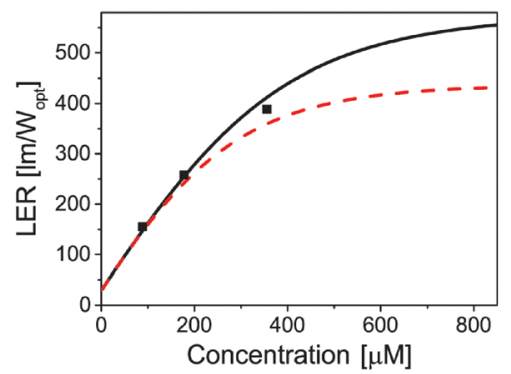

FIG. 2. Simulation and experimental comparison of color conversion for one type of FP (eGFP) on a blue electroluminescent LED. (a) Absorbance and photoluminescence of eGFP at a concentration level of $35.2 \mu \mathrm{M}$. (b) Theoretical and experimental absolute irradiance spectra of color conversion for a one type of FP at $88.4 \mu \mathrm{M}, 177.8 \mu \mathrm{M}$, and 355.6 $\mu \mathrm{M}$ eGFP concentration levels. (c) External quantum efficiency of eGFP. (d) Contribution of the respective absorption cycles to the photoluminescence spectra of $88.4 \mu \mathrm{M}, 177.8 \mu \mathrm{M}$, and $355.6 \mu \mathrm{M}$ eGFP concentration for $4 \mathrm{~mm}$ path length. (e) Peak electroluminescence wavelengths. (f) Peak photoluminescence wavelengths. (g) Luminous efficiency and (h) luminous efficacy of optical radiation. Red-dashed line: calculation without reabsorption, black line: calculation with reabsorption, black dots: experimental measurements. 
constant at the quantum yield of the fluorophore. This is because there is no radiative interaction that can generate energy losses for the emitted photons by the fluorophore. When we include the reabsorption process, the external quantum efficiency started to decrease with an increasing concentration of eGFP due to reabsorption [see Fig. 2(c)], which is in agreement with the experimental observations. We explored the reabsorption events that are responsible for the external quantum efficiency decay due to the effect of increasing concentration. For $177 \mu \mathrm{M}$ of eGFP, $78 \%$ of photoluminescence that is detected by the spectrophotometer is generated due to absorbance of the electroluminescent pump; whereas $17 \%$ is originating from the $1^{\text {st }}$ reabsorption cycle and $4 \%$ from the $2^{\text {nd }}$ reabsorption cycle [Fig. 2(d)]. As the number of reabsorption cycles increases, their contribution to the total emission decreases. When the concentration is increased to $355.6 \mu \mathrm{M}$, the contribution from the first and second reabsorption cycles increases to $21 \%$ and $6 \%$ due to the increased probability of photonic interactions, respectively.

We investigated the spectral peaks and observed a maximum of $2.6 \mathrm{~nm}$ blue shift of electroluminescence from the original peak position at $448.5 \mathrm{~nm}$ due to the higher absorption of $355 \mu \mathrm{M}$ eGFP concentration at longer wavelengths of electroluminescence [see Fig. 2(e)]. Moreover, we observed a maximum of $10.4 \mathrm{~nm}$ redshift of the eGFP photoluminescence peak from $510 \mathrm{~nm}$ due to reabsorption [see Fig. 2(f), black] for $355 \mu \mathrm{M}$, while there was no shift for the simulation without reabsorption [see Fig. 2(e), red]. The luminous efficacy of optical radiation and luminous efficiency are also in close agreement [Figs. 2(g) and 2(h)]. We found that the concentration of $355 \mu \mathrm{M}$ for eGFP yields a strong green photoluminescence [see Figs. S2(c) and S2(d)] (supplementary material), and above this concentration, the green photoluminescence and the luminous efficiency start to decrease due to the dominance of higher number of cycles.

\section{Simulation and experimental comparison of color conversion for a mixture of two types of fluorescent proteins}

A mixture of multiple types of fluorophore color mixing was analyzed to check the model. Now, the complexity is further increased when the fluorophore eGFP is used in conjunction with mCherry, which introduces scattering into a color conversion medium (see Fig. S2 of supplementary material). To include the scattering effect, we modified the Beer-Lambert law via the differential pathlength correction factor $(\gamma)$, which is used in tissue optics. ${ }^{23}$ The correction factor was fitted with the Rayleigh-type scattering of $\lambda^{-4}$ (see Fig. S2 of supplementary material), which yields the final form in Eq. (24). $c_{1}$ and $c_{2}$ were swept between [0.1, 4] and $[-1,1]$ to find the least root mean square error (RMSE) between simulated and experimental results for a mixture of 326 and $463 \mu \mathrm{M}$ for eGFP and mCherry, and the minima were obtained at 1.93 and -0.16 for $c_{1}$ and $c_{2}$, respectively a)



c)

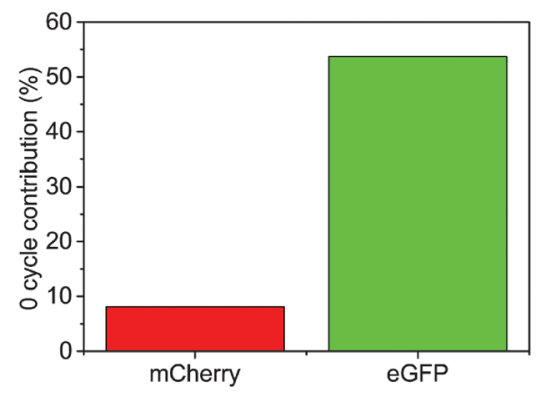

e)

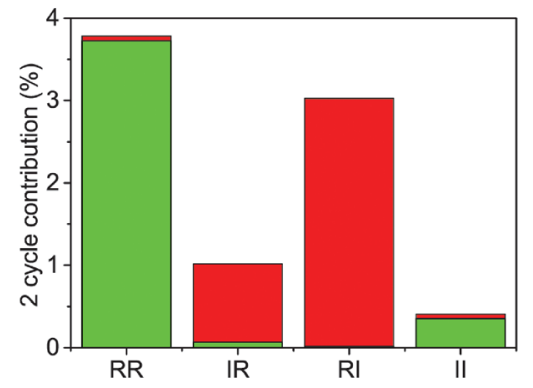

b)

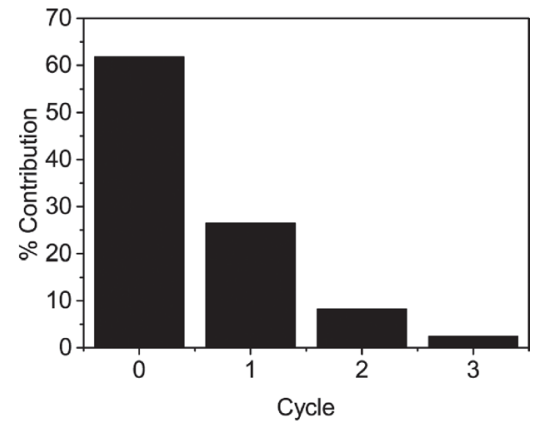

d)

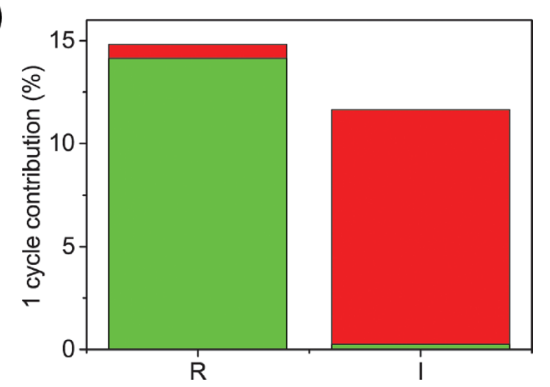

FIG. 3. Simulation and experimental comparison of color conversion for a mixture of two types of FPs (eGFP and mCherry) on blue electroluminescence. (a) Theoretical and experimental absolute irradiance spectra of color conversion for a mixture of two types of FPs. (b) Contribution of respective absorption cycles to the photoluminescence spectrum. (c)-(e) The photoluminescence contributions from zero, one, and two absorption cycles, respectively. 
(see Fig. S3 of supplementary material). This gave the spectral fit shown in Fig. 3(a). Since $\kappa$ factors are independent of the optical pathlength, $\kappa$ remains constant for eGFP and mCherry [see Eqs. (S17)-(S23) for the differential pathlength corrected general equation]

$$
\gamma(\lambda)=c_{1}+c_{2} R(\lambda)
$$

where $\mathrm{R}$ is Rayleigh scattering $\left(R(\lambda)=c \lambda^{-4}(\mathrm{~nm}), c=2.2\right.$ $\times 10^{11}$ ) (see Fig. S2 of supplementary material).

We extracted the contributing photonic processes for the generated spectrum. At $0^{\text {th }}$ cycle, where no reabsorption or inter-absorption occurs, the photoluminescence generated by eGFP and mCherry is solely due to direct absorption of the electroluminescent blue pump. In the mixture, eGFP has a higher $\kappa$ with respect to mCherry $\left(\kappa_{e G F P}=0.9, \kappa_{m \text { Cherry }}=0.1\right.$ at $450 \mathrm{~nm}$ ), thus eGFP more strongly absorbs electroluminescence of the blue pump. In addition, since the quantum yield of eGFP is higher than mCherry $\left(Q_{e G F P}=0.6, Q_{m \text { Cherry }}\right.$ $=0.22$ ), eGFP photoluminescence contributes $53.4 \%$, whereas mCherry photoluminescence contributes $7.4 \%$ to total photoluminescence due to direct absorption [see Fig. 3(c) and Table $\mathrm{S} 1$ of supplementary material].

The $1^{\text {st }}$ absorption cycle, where only a single event of either reabsorption or inter-absorption occurs, generates $25.5 \%$ of the total photoluminescence [see Fig. 3(d)]. The absorbance and photoluminescence overlap of eGFP facilitates significant reabsorption-originated emission $\left(M_{11}^{1}\right)$, which occupies $14.5 \%$ of the total photoluminescence [see Fig. 3(d)]. Since there is a significant overlap of photoluminescence of eGFP and absorbance of mCherry, inter-absorption has important role for the generation of mCherry photoluminescence $\left(M_{12}^{1}\right)$, which also has $10.1 \%$ contribution to total photoluminescence. Due to the lower quantum yield of mCherry, most of the reabsorbed mCherry photoluminescence has non-radiative recombination and only a small portion of the intensity $(0.6 \%)$ has radiative recombination $\left(M_{22}^{1}\right)$. Moreover, interestingly, the overlap of photoluminescence of mCherry and absorbance of eGFP also has a small contribution to the generated spectrum, which is due to the term $M_{21}^{1}$ with $0.2 \%$ contribution.

For the 2nd absorption cycle, there are two events of reabsorption or inter-absorption, which are iteratively reabsorption-reabsorption (RR), interabsorption-reabsorption (IR), reabsorption-interabsorption (RI), and interabsorptioninterabsorption (II). Because of the scaling effect with the quantum yield of the respective fluorophores, its contribution is around $7.7 \%$. The main contribution to total emission comes from the reabsorption-reabsorption originated luminescence by eGFP [see Fig. 3(d)].

\section{CONCLUSION}

In this study, we developed a new method that includes absorption, photoluminescence, reabsorption and interabsorption of color conversion for a mixture of multiple types of fluorophores. We measured and simulated output optical spectra for one and a mixture of two types of FPs, and the theoretical and experimental results show good agreement. Moreover, the method clearly identifies the responsible photonic processes that are observed in the output spectrum, which is impossible to resolve experimentally. Advantageously, the developed method is simple and it can be easily coded on mathematical softwares (e.g., MATLAB). The results can be quickly computed on a standard computer due to its basis on matrix algebra. Moreover, the method can be used for all types of fluorophores, and the efficiency limits with the existing fluorophores can be determined. At the same time, by using a top-down approach, the optimal spectral properties can be defined for application-specific light sources, and new materials can be designed according to the results obtained by the method. Therefore, this method has high potential to impact the material compositions, their integration for devices, and enhance the design and understanding of state-of-the-art and future optoelectronic devices.

\section{SUPPLEMENTARY MATERIAL}

See supplementary material for the derivation of the $\kappa$ factor, matrix elements for the radiative energy transfer interactions for the first three cycles, experimental setup, absolute irradiance of electroluminescence blue pump, color conversion spectra of one type of fluorescent protein, absorbance and photoluminescence of eGFP and mCherry, RMSE error between simulated and experimental output spectra and the contribution of eGFP and mCherry to the total photoluminescence of color conversion for a mixture of two types of FPs.

\section{ACKNOWLEDGMENTS}

The research leading to these results has received funding from Technological Research Council of Turkey (TUBITAK) with Project Nos. 114F317, 115E115, 115E242, 115F451, and 114E194, Marie Curie Career Integration Grant (PROTEINLED, 631679), and Turkish Academy of Sciences (TUBA). We thank the VESTEL Electronics, Inc., Manisa, Turkey, for providing the LED chips.

${ }^{1}$ S. Pimputkar, J. S. Speck, S. P. DenBaars, and S. Nakamura, "Prospects for LED lighting," Nat. Photonics 3(4), 180-182 (2009).

${ }^{2}$ S. E. Braslavsky, "Glossary of terms used in photochemistry 3(rd) edition (IUPAC Recommendations 2006)," Pure Appl. Chem. 79(3), 293-465 (2007).

${ }^{3}$ S. Tasch, C. Brandstatter, F. Meghdadi, G. Leising, G. Froyer, and L. Athouel, "Red-green-blue light emission from a thin film electroluminescence device based on parahexaphenyl," Adv. Mater. 9(1), 33 (1997).

${ }^{4}$ S. Tasch, E. J. W. List, O. Ekstrom, W. Graupner, G. Leising, P. Schlichting et al., "Efficient white light-emitting diodes realized with new processable blends of conjugated polymers," Appl. Phys. Lett. 71(20), 2883-2885 (1997).

${ }^{5}$ J. K. Sheu, S. J. Chang, C. H. Kuo, Y. K. Su, L. W. Wu, Y. C. Lin et al., "White-light emission from near UV InGaN-GaN LED chip precoated with blue/green/red phosphors," IEEE Photonics Technol. Lett. 15(1), 18-20 (2003).

${ }^{6}$ H. V. Demir, S. Nizamoglu, T. Erdem, E. Mutlugun, N. Gaponik, and A. Eychmuller, "Quantum dot integrated LEDs using photonic and excitonic color conversion," Nano Today 6(6), 632-647 (2011).

${ }^{7}$ D. A. Press, R. Melikov, D. Conkar, E. N. Firat-Karalar, and S. Nizamoglu, "Fluorescent protein integrated white LEDs for displays," Nanotechnology 27(45), 45LT01 (2016).

${ }^{8}$ M. Meyns, M. Peralvarez, A. Heuer-Jungemann, W. Hertog, M. Ibanez, R. Nafria et al., "Polymer-enhanced stability of inorganic perovskite nanocrystals and their application in color conversion LEDs," ACS Appl. Mater. Interfaces 8(30), 19579-19586 (2016). 
${ }^{9}$ L. B. Tang, R. B. Ji, X. K. Cao, J. Y. Lin, H. X. Jiang, X. M. Li et al., "Deep ultraviolet photoluminescence of water-soluble self-passivated graphene quantum dots," ACS Nano 6(6), 5102-5110 (2012).

${ }^{10}$ Protein Integrated White LEDs for Lighting. Novel Optical Materials and Applications, edited by D. A. Press, R. Melikov, D. Conkar, E. N. FiratKaralar, and S. Nizamoglu (Optical Society of America, 2016).

${ }^{11}$ R. Melikov, D. A. Press, B. G. Kumar, I. B. Dogru, S. Sadeghi, M. Chirea et al., "Silk-hydrogel lenses for light-emitting diodes," Sci. Rep.-Uk 7(1), 7258 (2017).

${ }^{12}$ J. M. Phillips, M. E. Coltrin, M. H. Crawford, A. J. Fischer, M. R. Krames, R. Mueller-Mach et al., "Research challenges to ultra-efficient inorganic solid-state lighting," Laser Photonics Rev. 1(4), 307-333 (2007).

${ }^{13}$ A. Zukauskas, R. Vaicekauskas, F. Ivanauskas, R. Gaska, and M. S. Shur, "Optimization of white polychromatic semiconductor lamps," Appl. Phys. Lett. 80(2), 234-236 (2002).

${ }^{14}$ T. Erdem, S. Nizamoglu, and H. V. Demir, "Computational study of power conversion and luminous efficiency performance for semiconductor quantum dot nanophosphors on light-emitting diodes," Opt. Express 20(3), 3275-3295 (2012).

${ }^{15}$ T. Erdem, S. Nizamoglu, X. W. Sun, and H. V. Demir, "A photometric investigation of ultra-efficient LEDs with high color rendering index and high luminous efficacy employing nanocrystal quantum dot luminophores," Opt. Express 18(1), 340-347 (2010).

${ }^{16}$ A. R. Duggal, J. J. Shiang, C. M. Heller, and D. F. Foust, "Organic lightemitting devices for illumination quality white light," Appl. Phys. Lett. 80(19), 3470-3472 (2002).
${ }^{17}$ J. S. Batchelder, A. H. Zewail, and T. Cole, "Luminescent solar concentrators. 1: Theory of operation and techniques for performance evaluation," Appl. Opt. 18(18), 3090-3110 (1979).

${ }^{18}$ J. S. Batchelder, A. H. Zewail, and T. Cole, "Luminescent solar concentrators. 2: Experimental and theoretical analysis of their possible efficiencies," Appl. Opt. 20(21), 3733-3754 (1981).

${ }^{19}$ W. Ji, L. Zhang, R. Gao, L. Zhang, W. Xie, H. Zhang et al., "Top-emitting white organic light-emitting devices with down-conversion phosphors: Theory and experiment," Opt. Express 16(20), 15489-15494 (2008).

${ }^{20} \mathrm{~W}_{\mathrm{A}}$ and $\mathrm{W}_{\mathrm{B}}$ is the amount of absorbed light by the fluorophore $\mathrm{A}$ and $\mathrm{B}$ from the electroluminescence and scaled by $\kappa_{\mathrm{A}}(\lambda)$ and $\kappa_{\mathrm{B}}(\lambda)$, which are ratio of absorbed light from the electroluminescence by individual fluorophores $\mathrm{A}$ and $\mathrm{B}$, and integrated over the spectrum and scaled by quantum yield of fluorophores $\mathrm{Q}_{\mathrm{A}}$ and $\mathrm{Q}_{\mathrm{B}}$; $\mathrm{f}_{\mathrm{XY}}$ is the amount of absorbed light by the fluorophore $\mathrm{X}$ from emission of fluorophore $\mathrm{Y}$ integrated over the spectrum and scaled by quantum yield of fluorophore $X(X=A, B)$.

${ }^{21}$ See http://www.tsienlab.ucsd.edu/Documents.htm for Spectra of Various Fluorescent Proteins [Internet] (2016)

${ }^{22}$ T. Asakura, Y. Watanabe, and T. Itoh, "Nmr of silk fibroin. 3. Assignment of carbonyl carbon resonances and their dependence on sequence and conformation in bombyx-mori silk fibroin using selective isotopic labeling," Macromolecules 17(11), 2421-2426 (1984).

${ }^{23} \mathrm{~F}$. Scholkmann and M. Wolf, "General equation for the differential pathlength factor of the frontal human head depending on wavelength and age," J. Biomed. Opt. 18(10), 1 (2013). 